\title{
Indices of Dependence Between Types in Multivariate Point Patterns
}

\author{
M. N. M. VAN LIESHOUT \\ CWI \\ A. J. BADDELEY \\ University of Western Australia
}

\begin{abstract}
We propose new summary statistics quantifying several forms of dependence between points of different types in a multi-type spatial point pattern. These statistics are the multivariate counterparts of the $J$-function for point processes of a single type, introduced by Lieshout \& Baddeley (1996). They are based on comparing distances from a type $i$ point to either the nearest type $j$ point or to the nearest point in the pattern regardless of type to these distances seen from an arbitrary point in space. Information about the range of interaction can also be inferred. Our statistics can be computed explicitly for a range of wellknown multivariate point process models. Some applications to bivariate and trivariate data sets are presented as well.
\end{abstract}

Key words: beta cells, bramble canes, empty space function, $J$-function, multi-type point patterns, myrtle disease, nearest-neighbour distance distribution function, random labelling, spatial interaction, spatial statistics

\section{Introduction}

A multivariate point pattern is a spatial pattern of points, each point belonging to one of a finite number of distinct types (Cox \& Lewis, 1972). Bivariate or two-type patterns in particular have often been reported and analysed. Examples considered in section 5 are a map of trees identified as healthy or diseased, a microscope image of retinal ganglion cells identified as "on" or "off", and a map of plants classified into three types according to age.

To investigate dependence between the different types of points, the usual approach (see e.g. Bartlett, 1975; Ripley, 1977; Diggle \& Cox, 1981; Lotwick \& Silverman, 1982; Diggle, 1983; Vincent \& Jeulin, 1989; Cressie, 1991; Stoyan et al., 1995) begins by estimating "cross-type" versions of the standard summary functions $G$ and $K$. The purpose of this paper is to pursue an alternative.

In a previous paper (Lieshout \& Baddeley, 1996), we introduced a new summary function $J$ for univariate (single-type) point patterns $X$ as

$$
J(t)=\frac{1-G(t)}{1-F(t)}
$$

defined for all $t \geqslant 0$ with $F(t) \neq 1$. Here the "empty space function" $F$ is the distribution function of the distance from an arbitrary fixed point 0 to the nearest point of the pattern $X$, and the "nearest neighbour distance function" $G$ is the distribution function of the distance from a typical point of $X$ to the nearest other point of $X$. The $J$-function is an index of spatial interaction, identically equal to 1 for a Poisson process, and generally takes values less than 1 for clustered patterns and greater than 1 for ordered patterns. An 
appealing property is that the superposition $X .=X_{1} \cup X_{2}$ of two independent point processes $X_{1}, X_{2}$ has $J$-function

$$
J(t)=\frac{\lambda_{1}}{\lambda_{1}+\lambda_{2}} J_{1}(t)+\frac{\lambda_{2}}{\lambda_{1}+\lambda_{2}} J_{2}(t)
$$

where $J_{1}, J_{2}$ are the $J$-functions of $X_{1}, X_{2}$ respectively and $\lambda_{1}, \lambda_{2}$ are their intensities. A similar statement holds for the superposition of $m$ independent point processes.

In the present paper we extend these ideas to multivariate point patterns. Let $X_{i}$ be the process of type $i$ points and $X .=X_{1} \cup \cdots \cup X_{m}$ the process of all points regardless of type. Three approaches are proposed, which correspond to investigating three different forms of independence between types. First we may compare the left and right hand sides of (2) or its analogue for $m$ types. These two expressions are equal if $X_{1}, \ldots, X_{m}$ are independent. Secondly we may construct an "inter-type" $J$-function $J_{i j}$ for each pair of types $i$ and $j$. This function is identically equal to 1 if $X_{i}$ and $X_{j}$ are (marginally) independent. Thirdly we may construct a function $J_{i}$. for each $i$ summarizing the dependence of $X$. on $X_{i}$. This reduces to a simple form if $X_{i}$ is independent of $\left(X_{j}, j \neq i\right)$, and to another simple form if the "random labelling" property holds.

Section 2 contains preliminaries and the main definitions of the $J$-functions; in section 3 a selection of basic properties including mixture formulas, representations in terms of conditional intensities and their importance to the interaction range, and behaviour under various independence assumptions are considered. In section 4 we calculate the $J$-functions for a wide variety of stochastic models and section 5 contains applications to bivariate and trivariate point pattern data.

\section{Definitions and notation}

\subsection{Univariate J-function}

First we recall some definitions from Lieshout \& Baddeley (1996). Throughout this paper we will assume that $X$ is a stationary point process in $\mathbb{R}^{d}$ with finite positive intensity $\lambda$, in order to allow for non-parametric inference based on a single observation of $X$. Then, the empty space function $F$ of $X$ is the cumulative distribution function of the distance from a fixed point (say, the origin) to the nearest point of $X$. Thus for $t \geqslant 0$

$$
F(t)=\mathbb{P}\{X \cap B(0, t) \neq \varnothing\}
$$

where $B(0, t)$ is the closed ball of radius $t$ centred at the origin 0 . The nearest neighbour distance function $G$ is the distribution of the distance from a typical point of $X$ to the nearest other point of $X$. For $t \geqslant 0$

$$
\begin{aligned}
G(t) & =P^{0}\{X \cap B(0, t) \backslash\{0\} \neq \varnothing\} \\
& =P^{!(0)}\{X \cap B(0, t) \neq \varnothing\}
\end{aligned}
$$

where $P^{()}$is the Palm distribution (Kallenberg, 1984; Daley \& Vere-Jones, 1988; Stoyan et al., 1995) of $X$ at 0 , which can be interpreted as the conditional distribution of $X$ given that there is a point of $X$ at 0 . On the last line $P^{! 0}$ is the reduced Palm distribution, defined as the Palm distribution of the process with the point at 0 removed, i.e. $P^{! 0}$ is the distribution of $X \backslash\{0\}$ under $P^{()}$.

In an earlier paper (Lieshout \& Baddeley, 1996) we introduced

$$
J(t)=\frac{1-G(t)}{1-F(t)}
$$


defined for all $t \geqslant 0$ with $F(t)<1$. If $X$ is a Poisson process then $J \equiv 1$. We found several representations of $J$ derived from the Nguyen-Zessin formula (Nguyen \& Zessin, 1979)

$$
\lambda \mathbf{E}^{! 0}[f(X)]=\mathbb{E}[\lambda(0 ; X) f(X)]
$$

where $f$ is any non-negative measurable function on the space of realizations of $X, \mathbf{E}^{10}$ denotes the expectation with respect to $P^{! 0}$, and $\lambda(0 ; X)$ is the conditional intensity of $X$ at 0 assuming this exists.

\subsection{Multivariate J-functions}

Throughout the present paper we consider a stationary multivariate point pattern $Y$ in $\mathbb{R}^{d}$, each point belonging to one of $m$ types. Formally $Y$ is a stationary marked point process in $\mathbb{R}^{d}$ with marks in $\{1,2, \ldots, m\}$. Equivalently $Y=\left(X_{1}, \ldots, X_{m}\right)$ is an $m$-tuple of jointly stationary point processes in $\mathbb{R}^{d}$, where $X_{i}$ is the process consisting of points of type $i$. Write $\lambda_{i}$ for the intensity of $X_{i}$. Define

$$
X=\bigcup_{i=1}^{m} X_{i}
$$

the point process consisting of all random points regardless of type.

Henceforth $i$ and $j$ denote indices from the set $\{1, \ldots, m\}$. Let $F_{i}$ and $F$. be the empty space functions of $X_{i}$ and $X$. respectively; thus for $t \geqslant 0$

$$
\begin{aligned}
& F_{i}(t)=\mathbb{P}\left\{X_{i} \cap B(0, t) \neq \varnothing\right\}, \\
& F .(t)=\mathbb{P}\{X . \cap B(0, t) \neq \varnothing\} .
\end{aligned}
$$

Let $P^{(0, i)}$ be the Palm distribution of $Y$ conditional on a point at 0 with mark $i$, and $P^{!(0, i)}$ the corresponding reduced Palm distribution, i.e. the distribution of $Y \backslash\{(0, i)\}$ under $P^{(0, i)}$. Then define the " $i$-to- $j$ " nearest neighbour distance function

$$
G_{i j}(t)=P^{!(0, i)}\left\{X_{j} \cap B(0, t) \neq \varnothing\right\}
$$

and the " $i$-to-any" nearest neighbour distance function

$$
G_{i .}(t)=P^{!(0, i)}\{X, \cap B(0, t) \neq \varnothing\} .
$$

Thus $G_{i j}$ is the distribution function of the distance from a typical point of type $i$ to the nearest point of type $j$, and $G_{i}$. from a typical point of type $i$ to the nearest point of any type. To keep notation uniform we write $G$.. for the ordinary $G$ function of $X$.

\section{Definition 1}

For a stationary multivariate point process $\left(X_{1}, \ldots, X_{m}\right)$ on $\mathbb{R}^{d}$ define $($ for $i, j=1, \ldots, m$ )

$$
\begin{aligned}
& J_{i j}(t)=\frac{1-G_{i j}(t)}{1-F_{j}(t)} \\
& J_{i .}(t)=\frac{1-G_{i .}(t)}{1-F_{.}(t)}
\end{aligned}
$$

for all $t \geqslant 0$ for which $F_{j}(t)<1$ or $F .(t)<1$ respectively. For uniformity of notation we will also write

$$
J_{. .}(t)=\frac{1-G_{. .}(t)}{1-F_{.}(t)}
$$

for the $J$-function of $X$. 
In particular $J_{i i}$ is the $J$-function of the univariate process $X_{i}$. Note that the definition of $J_{i j}$ is not symmetric in $i$ and $j$. While this may be undesirable for inference on processes appearing on an equal footing, it may be easier to interpret, especially when considering qualitatively different patterns.

Intuitively $J_{i j}$ is a comparison between the distributions of the distances to the nearest type $j$ point, measured from (a) an arbitrary fixed point in $\mathbb{R}^{d}$, and (b) a typical type $i$ point. The denominator of (4) is the unconditional probability of the event that there is no type $j$ point within a distance $t$ of 0 . The numerator is the "conditional probability" of the same event given that there is a type $i$ point at 0 .

As in the univariate case (Lieshout \& Baddeley, 1996) the value 1 is obtained when there is no spatial interaction: if $X_{i}$ and $X_{j}$ are independent processes, then standard calculations give $J_{i j} \equiv 1$. However, having a $J_{i j}$-function taking value 1 everywhere should not be seen as a characterization of independence. In particular $J_{i i} \equiv 1$ is not a sufficient condition for $X_{i}$ to be a Poisson process (Bedford \& van den Berg, 1997). Similar remarks apply to the other statistics introduced in definition 1 .

Values $J_{i j}>1$ can be interpreted as indicating inhibition (of type $j$ points by type $i$ points) since this is equivalent to $G_{i j}<F_{j}$, i.e. the presence of a type $i$ point decreases the probability of finding a type $j$ point nearby. Similarly, values less than 1 suggest positive association.

The statistic $J_{i}$. is a comparison between the distributions of the distances to the nearest random point of any type, measured from the origin and from a type $i$ point. An interpretation of the values of $J_{i}$ analogous to that of $J_{i j}$ applies. If $\left(X_{1}, \ldots, X_{m}\right)$ are independent then $J_{i .}(t)=J_{i i}(t)$, the marginal J-function. If furthermore $X_{i}$ is a Poisson process, then $J_{i .}(t) \equiv 1$.

With equation (2) in mind we introduce the following function $I$.

\section{Definition 2}

For a stationary multivariate point process $\left(X_{1}, \ldots, X_{m}\right)$ on $\mathbb{R}^{d}$ define

$$
I(t)=\sum_{i=1}^{m} \frac{\lambda_{i}}{\lambda} J_{i i}(t)-J_{. .}(t)
$$

for all $t \geqslant 0$ with $F_{.}(t)<1$ and where $\lambda_{i}$ is the intensity of $X_{i}$ and $\lambda .=\sum_{i=1}^{m} \lambda_{i}$ is the intensity of $X$.

If $X_{1}, \ldots, X_{m}$ are independent then $I \equiv 0$ by th. 2 in Lieshout \& Baddeley (1996). Note that in the definition of $I$ all component processes appear on an equal footing.

At least for bivariate processes $Y=\left(X_{1}, X_{2}\right)$, the sign of $I(t)$ should indicate the type of association between the two components $X_{1}, X_{2}$ with a positive value being suggestive of positive dependence. This definition is similar to Lotwick \& Silverman's (1982) suggestion of studying the sign of

$$
T(t)=\log \left(1-F_{.}(t)\right)-\log \left(1-F_{1}(t)\right)-\log \left(1-F_{2}(t)\right)
$$

$(t \geqslant 0)$ for a bivariate point process, since $T$ is identically zero when $X_{1}$ and $X_{2}$ are independent point processes.

\section{Basic properties}

\subsection{Mixture formulas}

\section{Lemma 1}

For any stationary multivariate point process $Y=\left(X_{1}, \ldots, X_{m}\right)$

(1) Board of the Foundation of the Scandinavian Journal of Statistics 1999. 


$$
\begin{aligned}
& G_{. .}(t)=\sum_{i=1}^{m} \frac{\lambda_{i}}{\lambda} G_{i .}(t) \\
& J_{. .}(t)=\sum_{i=1}^{m} \frac{\lambda_{i}}{\lambda} J_{i .}(t) \\
& I(t)=\sum_{i=1}^{m} \frac{\lambda_{i}}{\lambda}\left[J_{i i}(t)-J_{i .}(t)\right]
\end{aligned}
$$

for all $t \geqslant 0$ with $F .(t)<1$.

Proof. The reduced Palm distribution at 0 of $Y$ with respect to $X$. is

$$
\sum_{i=1}^{m} \frac{\lambda_{i}}{\lambda} P^{!(0, i)} .
$$

This yields the expression for $G$... The remaining identities follow by substitution.

Mixture formulas are useful from a computational point of view when dealing with patterns consisting of a large number of points, since they offer a natural way of breaking up the necessary distance calculations needed for $G_{. .}, J_{\text {.. }}$ or $I$ in more manageable parts.

\subsection{Case of independence}

Here we calculate the multivariate $J$-functions when some form of independence holds between types. In particular, full independence and pairwise independence are considered, as well as independence between one of the components and the vector or union of the other components of $Y$. Finally, a random labelling property is considered, which may be more appropriate in cases where the type labels are assigned after the locations have been occupied (see the examples discussed in section 5).

\section{Lemma 2}

Let $i \neq j$. If $X_{i}$ and $X_{j}$ are (marginally) independent then $J_{i j} \equiv 1$ where defined.

Proof. Clearly $J_{i j}$ depends only on the marginal joint distribution of $\left(X_{i}, X_{j}\right)$. If $X_{i}, X_{j}$ are independent then the distribution of $X_{j}$ under $P^{!(0, i)}$ is the same as its ordinary marginal distribution, so $G_{i j} \equiv F_{j}$ (see e.g. Diggle, 1983, p. 92; or Cressie, 1991, p. 700) hence $J_{i j} \equiv 1$.

\section{Lemma 3}

If $X_{i}$ is independent of $\left(X_{j}, j \neq i\right)$, then $J_{i .} \equiv J_{i i}$ where defined. More generally this holds if $X_{i}$ is independent of $X_{-i}=\bigcup_{j \neq i} X_{j}$, the univariate process consisting of points of all types except $i$.

Proof. Let $F_{-i}$ be the empty space function of $X_{-i}$. If $X_{i}$ and $X_{-i}$ are independent, then

$$
1-F_{.}(t)=\left(1-F_{i}(t)\right)\left(1-F_{-i}(t)\right)
$$

and under $P^{!(0, i)}, X_{i}$ and $X_{-i}$ are also independent with $X_{i}$ governed by its reduced Palm distribution at 0 , and $X_{-i}$ by its ordinary marginal distribution. Thus

$$
1-G_{i .}(t)=\left(1-G_{i i}(t)\right)\left(1-F_{-i}(t)\right)
$$

and division by $1-F_{.}(t)$ yields the result. 


\section{Definition 3}

The marked point process $Y$ has the random labelling property if the marks (types) of the points are conditionally i.i.d. given the locations of the points.

For example, if $Y=\left(X_{1}, \ldots, X_{m}\right)$ for independent Poisson processes $X_{i}, i=1, \ldots, m$, then $Y$ has the random labelling property. In general, however, independence and random labelling are not equivalent. An informal treatment of the distinction between these two types of independence assumptions with particular attention to their implications in practice is given by Diggle (1983, sect. 6.3).

\section{Lemma 4}

Under the random labelling assumption with label probabilities $p_{i}(i=1, \ldots, m)$, for all $t \geqslant 0$ with $F .(t)<1$

$$
J_{i .}(t)=J_{. .}(t)
$$

while whenever $F_{j}(t)<1$

$$
J_{i j}(t)=\frac{\mathbb{E}^{10}\left[\left(1-p_{j}\right)^{N_{t}}\right]}{\mathbb{E}\left[\left(1-p_{j}\right)^{N_{t}}\right]}
$$

where $N_{t}=N(X, \cap B(0, t))$ is the number of points of any type in $B(0, t)$, and $\mathbb{E}^{! 0}$ denotes expectation with respect to the reduced Palm distribution of $X$.

Proof. If $Q$ is the distribution of any univariate point process $Z$, let $\rho(Q)$ denote the distribution of the multivariate point process obtained by assigning i.i.d. random marks to the points of $Z$.

The random labelling assumption is that $P=\rho(P$.) where $P$ is the distribution of $\left(X_{1}, \ldots, X_{m}\right)$ and $P$. is the distribution of $X$. It can easily be shown that under random labelling,

$$
p^{!(0, i)}=\rho\left(P^{! 0}\right)
$$

i.e. the reduced Palm distribution of $\left(X_{1}, \ldots, X_{m}\right)$ given a point of type $i$ at 0 is equivalent to applying random labelling to the reduced Palm distribution of $X$. Thus

$$
\begin{aligned}
G_{i .}(t) & =P^{!(0, i)}\{X \cap \cap(0, t) \neq \varnothing\} \\
& =\rho\left(P_{.}^{! 0}\right)\{X . \cap B(0, t) \neq \varnothing\} \\
& =P^{! 0}\{X . \cap B(0, t) \neq \varnothing\} \\
& =G_{. .}(t)
\end{aligned}
$$

and substituting in the definition of $J_{i}$. yields (11). The second result (12) follows from (13) and the representation of the $J$-function for an independent thinning (Lieshout \& Baddeley, 1996, th. 3).

\subsection{Representations}

Here we investigate explicit representations for the various $J$ - and $I$-functions in terms of conditional intensities, analogous to the univariate case (Lieshout \& Baddeley, 1996, th. 1).

Write $\lambda_{i}\left(0 ; X_{i}\right)$ for the conditional intensity of $X_{i}$ at 0 , and $\lambda .(0 ; X$.) for that of $X$, defined to satisfy the analogues of the Nguyen-Zessin formula (3), if they exist. Let $\lambda((0, i) ; Y)$ be the

(C) Board of the Foundation of the Scandinavian Journal of Statistics 1999. 
conditional intensity (if it exists) of the multivariate process $Y=\left(X_{1}, \ldots, X_{m}\right)$ for a point at 0 with type $i$, defined to satisfy the multivariate counterpart of (3)

$$
\lambda_{i} \mathbb{E}^{!(0, i)} f(Y)=\mathbb{E}[\lambda((0, i) ; Y) f(Y)]
$$

for any non-negative measurable function $f$ on the space of realizations of $Y$. In particular taking $f \equiv 1$

$$
\lambda_{i}=\mathbb{E} \lambda((0, i) ; Y) .
$$

The existence of $\lambda((0, i) ; Y)$ implies that of $\lambda_{i}\left(0 ; X_{i}\right)$ and $\lambda_{(}\left(0 ; X_{\text {. }}\right)$ and indeed

$$
\begin{aligned}
& \mathbb{E}\left[\lambda((0, i) ; Y) \mid X_{i}\right]=\lambda_{i}\left(0 ; X_{i}\right) \text { a.s. } \\
& \mathbb{E}\left[\sum_{i=1}^{m} \lambda((0, i) ; Y) \mid X .\right]=\lambda .(0 ; X .) \text { a.s. }
\end{aligned}
$$

by (3) and (14).

\section{Lemma 5}

Let $Y=\left(X_{1}, \ldots, X_{m}\right)$ be any stationary multivariate point process for which the conditional intensity $\lambda((0, i) ; Y)$ exists and satisfies (14).

Then $G_{i j}(t)<1$ implies $F_{j}(t)<1$ and

$$
\begin{aligned}
J_{i j}(t) & =\mathbb{E}\left[\frac{\lambda((0, i) ; Y)}{\lambda_{i}} \mid X_{j} \cap B(0, t)=\varnothing\right] \\
& =\left(\mathbb{E}^{!(0, i)}\left[\frac{\lambda_{i}}{\lambda((0, i) ; Y)} \mid X_{j} \cap B(0, t)=\varnothing\right]\right)^{-1} .
\end{aligned}
$$

Similarly $G_{i .}(t)<1$ implies $F(t)<1$ and

$$
\begin{aligned}
J_{i .}(t) & =\mathbb{E}\left[\frac{\lambda((0, i) ; Y)}{\lambda_{i}} \mid X . \cap B(0, t)=\varnothing\right] \\
& =\left(\mathbb{E}^{(!(0, i)}\left[\frac{\lambda_{i}}{\lambda((0, i) ; Y)} \mid X . \cap B(0, t)=\varnothing\right]\right)^{-1} .
\end{aligned}
$$

Expressions for $I(t)$ can be obtained by substituting (17)-(20) in (9).

These results should be compared to similar expressions in the univariate case, see Lieshout \& Baddeley, 1996, th. 1 .

Proof. For (17)-(18), use the Nguyen-Zessin formula (14) taking $f(Y)=$ $\mathbf{1}\left\{X_{j} \cap B(0, t)=\varnothing\right\}$ or $f(Y)=\mathbf{1}\left\{X_{j} \cap B(0, t)=\varnothing\right\} / \lambda((0, i) ; Y)$. For (19)-(20) take $f(Y)=$ $\mathbf{1}\{X . \cap B(0, t)=\varnothing\}$ or $f(Y)=\mathbf{1}\{X . \cap B(0, t)=\varnothing\} / \lambda((0, i) ; Y)$.

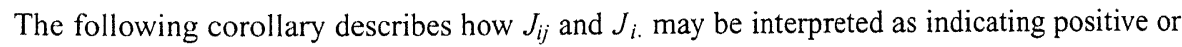
negative correlation between types, cf. Lieshout \& Baddeley, 1996, corall. 1, eq. (3.7).

\section{Lemma 6}

Let $Y=\left(X_{1}, \ldots, X_{m}\right)$ be any stationary multivariate point process for which the conditional intensity $\lambda((0, i) ; Y)$ exists and satisfies (14). Then $J_{i j}(t) \geqslant 1$ iff

$$
\operatorname{cov}\left(\lambda((0, i) ; Y), \mathbf{1}\left\{X_{j} \cap B(0, t)=\varnothing\right\}\right) \geqslant 0
$$




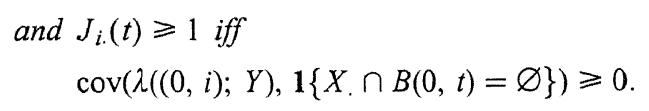

Proof. Rewriting (17) as

$$
J_{i j}(t)=\frac{\operatorname{cov}\left(\lambda((0, i) ; Y), \mathbf{1}\left\{X_{j} \cap B(0, t)=\varnothing\right\}\right)}{\lambda_{i} \mathbb{P}\left(X_{j} \cap B(0, t)=\varnothing\right)}+1
$$

we obtain the first result. Similarly for the second.

Using lemma 5 and the decomposition (9) we find that a sufficient condition for $I(t) \geqslant 0$ is that for all $i$,

$$
\mathbb{E}\left[\lambda_{i}\left(0 ; X_{i}\right) \mid X_{i} \cap B(0, t)=\varnothing\right] \geqslant \mathbb{E}[\lambda((0, i) ; Y) \mid X . \cap B(0, t)=\varnothing] .
$$

Reversing the signs gives a similar, sufficient condition for $I(t) \leqslant 0$.

\subsection{Finite interaction range}

Here we derive multivariate versions of th. 1(b) in Lieshout \& Baddeley (1996) stating that the $J$-function is constant for all $t \geqslant s$ if the process has finite interaction range $s$.

As in that paper, a univariate point process $X$ has interaction range $s, 0<s<\infty$ (or strictly speaking interaction range less than or equal to $s)$, if its conditional intensity $\lambda_{X}(0 ; X)$ is constant for all patterns $X$ which contain no points in $B(0, s)$. That is, $X \cap B(0, s)=\varnothing$ implies $\lambda_{X}(0 ; X)=\lambda_{X}(0 ; \varnothing)$.

\section{Definition 4}

A multivariate point process $Y=\left(X_{1}, \ldots, X_{m}\right)$ has joint interaction range $s$ if for each $i$, its multivariate conditional intensity $\lambda((0, i) ; Y)$ is constant for all realizations which contain no points in $B(0, s)$. That is, $X_{i} \cap B(0, s)=\varnothing$ for all $i$ implies $\lambda((0, i) ; Y)=\lambda((0, i) ; \varnothing)$.

A sufficient condition is that $\lambda((0, i) ; Y)$ depend only on $Y \cap B(0, s)$, the restriction of $Y$ to $B(0, s)$.

\section{Lemma 7}

If $Y$ has joint interaction range $s, 0<s<\infty$, then $J_{i .}(t)$ is constant for $t \geqslant s$,

$$
J_{i .}(t) \equiv \frac{\lambda((0, i) ; \varnothing)}{\lambda_{i}}, \quad t \geqslant s .
$$

If additionally the marginal processes $X_{i}$ each have interaction range $s$, then $I(t)$ is constant for $t \geqslant s$,

$$
I(t) \equiv \frac{1}{\lambda} \sum_{i=1}^{m}\left[\lambda_{i}(0 ; \varnothing)-\lambda((0, i) ; \varnothing)\right], \quad t \geqslant s
$$

or equivalently

$$
I(t) \equiv \frac{\sum_{i=1}^{m} \lambda_{i}(0 ; \varnothing)}{\sum_{i=1}^{m} \lambda_{i}}-\frac{\lambda .(0 ; \varnothing)}{\lambda .}, \quad t \geqslant s .
$$

An analogous statement for $J_{i j}$ does not hold in general.

(C) Board of the Foundation of the Scandinavian Journal of Statistics 1999. 
Proof. In (19) observe that $X . \cap B(0, t)=\varnothing$ for $t \geqslant s$ implies $X_{i} \cap B(0, s)=\varnothing$ for all $i$ so that $\lambda((0, i) ; Y)$ is conditionally constant and equal to $\lambda((0, i) ; \varnothing)$. The first result $(21)$ follows. The second result is proved by combining (21) with (9) and th. 1(b) in Lieshout \& Baddeley, (1996). The third result follows using (16) and the fact that $\lambda((0, i) ; Y)$ is conditionally constant.

\section{Theoretical examples}

In this section we calculate the multivariate $J$ functions for a variety of multivariate point pattern models.

\subsection{Multitype cluster processes}

By a multivariate cluster process in $\mathbb{R}^{d}$ we mean a general cluster process (Daley \& VereJones, 1988) constructed from a univariate point process in $\mathbb{R}^{d}$ (of "parent" points) by associating with each parent a cluster (of "offspring" points) which is a finite multivariate point process, i.e. a finite point process in $\mathbb{R}^{d} \times\{1, \ldots, m\}$. Only the offspring points are observed, but note that this is no restriction, since parents can be regarded as their own offspring.

We shall consider only the stationary multivariate Poisson cluster process in which the parents are a stationary Poisson process in $\mathbb{R}^{d}$ and, for a parent located at $x \in \mathbb{R}^{d}$, the cluster $Z_{x}$ of offspring of $x$ is distributed as $Z+x$, the vector translation by $x$ of a given, a.s. finite, multivariate point process $Z$. Offspring clusters from different parents $x_{i}$ are independent. Thus $Y=\bigcup_{i} Z_{x_{i}}$

\subsubsection{General result}

We need the multivariate version of a basic identity for cluster processes, see Bartlett (1975, pp. 8-9) as well as Daley \& Vere-Jones (1988) or Stoyan et al. (1995, p. 143). The functional form of the Palm distribution involved for a variety of univariate cluster processes is studied by Saxl \& Rataj (1996).

\section{Lemma 8}

Let $Y=\left(X_{1}, \ldots, X_{m}\right)$ be a stationary multivariate Poisson cluster process in $\mathbb{R}^{d}$. Then the Palm distribution of $Y$ with respect to a point of type $i$ at 0 can be written

$$
P^{(0, i)}=C^{(0, i)} * P
$$

where $P$ is the distribution of $Y, *$ denotes convolution and $C^{(0, i)}$ is the Palm distribution of the typical cluster with respect to a point of type $i$ at 0 . Similarly for the reduced Palm distributions,

$$
P^{!(0, i)}=C^{!(0, i)} * P \text {. }
$$

The following is a trivial corollary.

\section{Lemma 9}

For a stationary multivariate Poisson cluster process,

$$
\begin{aligned}
& J_{i j}(t)=C^{!(0, i)}\left\{Z_{j} \cap B(0, t)=\varnothing\right\} \\
& J_{i .}(t)=C^{!(0, i)}\{Z \cap \cap B(0, t)=\varnothing\}
\end{aligned}
$$

where $Z_{j}$ denotes the finite point process of points of type $j$ in the cluster, and $Z$. the process of all points in the cluster regardless of type. 
Thus, all $J_{i j}$ and $J_{i \text {. }}$-functions are decreasing and bounded above by 1 . Moreover $J_{i \text {. }} \leqslant J_{i i}$, suggesting clustered behaviour.

Regarding the range of interaction, if all clusters have maximum diameter $s$ then $J_{i j}(t)$ and $J_{i .}(t)$ are constant for all $t \geqslant s$.

\subsubsection{Two-type Gauss-Poisson process}

A Gauss-Poisson process (Bol'shakov, 1969; Newman, 1970; Milne \& Westcott, 1972) is a (univariate) Poisson cluster process in which each cluster consists either of one point (with probability $1-p$ ) or two points (with probability $p$ ). If a cluster has two points, they are separated by a random vector displacement $V$ which has probability density $h$ on $\mathbb{R}^{d}$.

Here we study the associated bivariate point process in which parent points are labelled as being of type 1 and daughter points, type 2 . This is a multivariate Poisson cluster process.

\section{Lemma 10}

For the two-type Gauss-Poisson process as described above,

$$
\begin{aligned}
& J_{11}(t)=J_{22}(t)=1 \\
& J_{12}(t)=J_{1 .}(t)=1-p H(t) \\
& J_{21}(t)=J_{2 .}(t)=1-H(t) \\
& J_{. .}(t)=1-\frac{2 p}{1+p} H(t) \\
& I(t)=\frac{2 p}{1+p} H(t)
\end{aligned}
$$

where

$$
H(t)=\int_{B(0, t)} h(x) d x
$$

Thus, the types 1 and 2 in this process are positively associated, in the senses measured by $J_{i i}$, $J_{i j}, J_{\text {.. }}$ and $I$. All the $J$-functions are decreasing and less than or equal to 1 (since $h$ is a probability density); $I$ is increasing and non-negative.

Furthermore, $J_{i} \leqslant J_{i i}$, suggesting positive association as well. However, regarding association between labels, for $p \notin\{0,1\}$,

$$
J_{2 .}(t)-J_{. .}(t)=\frac{p-1}{p+1} H(t)<0
$$

and decreasing, suggesting that the 2 to any distances appear more clustered than if the type is disregarded, while

$$
J_{1 .}(t)-J_{. .}(t)=\frac{p(1-p)}{p+1} H(t)>0
$$

and increasing, indicating that distances from a type 1 point appear more regular. A possible explanation is that any type 2 point will have a parent type 1 point, while a type 1 point may not have an associated type 2 point.

If we assume that $h$ is concentrated on a ball $B(0, R)$, then $J_{i j}(t), J_{i .}(t), J_{. .}(t)$ and $I(t)$ are constant for all $t \geqslant R$, mirroring the results for a univariate Poisson cluster process of Lieshout \& Baddeley (1996).

Proof. Both $X_{1}$ and $X_{2}$ are stationary Poisson processes, so $J_{i i} \equiv 1$.

(C) Board of the Foundation of the Scandinavian Journal of Statistics 1999. 
Under the Palm distribution $C^{(0,1)}$, with probability $1-p$ the cluster $Z$ has only a single point (of type 1 at 0 ), while with probability $p$ it has two points (one of type 1 at 0 and one of type 2 at $V$, where $V$ is random with density $h$ ). Applying lemma 9 ,

$$
\begin{aligned}
J_{12}(t) & =C^{!(0,1)}\left\{Z_{2} \cap B(0, t)=\varnothing\right\} \\
& =(1-p)+p \mathbb{P}\{V \notin B(0, t)\} \\
& =(1-p)+p(1-H(t)) \\
& =1-p H(t) .
\end{aligned}
$$

The calculation for $J_{1}$. produces the same result since $Z_{2} \equiv Z$. under $C^{!(0,1)}$.

Under $C^{(0,2)}$, with probability 1 the cluster has two points (of type 2 at 0 and of type 1 at $-V$ ). Applying lemma 9,

$$
\begin{aligned}
J_{21}(t) & =C^{!(0,2)}\left\{Z_{1} \cap B(0, t)=\varnothing\right\} \\
& =\mathbb{P}\{-V \notin B(0, t)\} \\
& =1-H(t) .
\end{aligned}
$$

Again the calculation for $J_{2}$. is identical.

Regarding $J_{. .}$, we apply the univariate version of lemma 8 . Now $C^{0}$ is number weighted. hence

$$
\begin{aligned}
J_{. .}(t) & =C^{0}\{Z \cap \cap B(0, t)=\{0\}\} \\
& =\frac{1-p}{1+p}+\frac{2 p}{1+p}(1-H(t)) .
\end{aligned}
$$

The result for $I$ follows using lemma 1 .

\subsection{Bivariate Poisson processes}

A bivariate Poisson process is a two-type process in which the marginal distribution of each of the components is that of a stationary Poisson process (Griffiths \& Milne, 1978; Diggle, 1983; Cressie, 1991).

A linked Poisson process (Diggle \& Cox, 1981) is a bivariate Poisson cluster process in which every cluster consists of exactly two points, a type 1 point and a type 2 point, separated by a random displacement $V$ where $V$ has density $h$ on $\mathbb{R}^{d}$. This is the special case $p=1$ of our twotype Gauss-Poisson process, so lemma 10 can be applied and we obtain $J_{11} \equiv J_{22} \equiv 1$, $J_{12}(t)=J_{21}(t)=J_{1 .}(t)=J_{2 .}(t)=J_{. .}(t)=1-H(t)$ and $I(t)=H(t)$. In particular, $J_{i} . \equiv J$. $(i=1,2)$, so the model is consistent with a random labelling assumption. However, in general, a randomly labelled linked Poisson process is not a linked Poisson process which can be seen easily if the displacement $V$ is taken to be deterministic.

Clearly, linked Poisson models exhibit positive dependence between the components, as confirmed by the expressions of the various $J$ - and $I$-functions computed above. For bivariate Poisson processes with negative dependence, see for instance Brown et al. (1981).

\subsection{Bivariate Cox processes}

A bivariate Cox process (Cox \& Lewis, 1972; Diggle \& Milne, 1983) is formed as follows. We start with two random measures $\Lambda_{1}, \Lambda_{2}$ on $\mathbb{R}^{d}$ which are typically dependent. Conditional on $\left(\Lambda_{1}, \Lambda_{2}\right)=\left(\lambda_{1}, \lambda_{2}\right)$, let $X_{1}$ and $X_{2}$ be independent inhomogeneous Poisson pro cesses with intensity measures $\lambda_{1}$ and $\lambda_{2}$ respectively. Then the unconditional model $Y$ $\left(X_{1}, X_{2}\right)$ is a bivariate Cox process. 


\subsubsection{Linked bivariate Cox process}

As an example of positive dependence consider the case where $\Lambda_{1}=\nu \Lambda_{2}$ for some fixed positive constant $v$; the resulting $Y$ is called a linked bivariate Cox process (Cox \& Lewis, 1972).

\section{Lemma 11}

Let $\left(X_{1}, X_{2}\right)$ be a linked Cox process where the intensity measures are "mixed Poisson", $\Lambda_{2}=A m$, for some non-negative random variable $A$ with finite positive expectation and where $m$ is Lebesgue measure. Write $L(s)=\mathbb{E} \exp (-s A)$ for the moment generating function of $A$. Then $\mathbb{E}[A \exp (-s A)]=-L^{\prime}(s)$ and

$$
\begin{aligned}
J_{11}(t) & =J_{21}(t)=L^{\prime}\left(v \kappa_{d} t^{d}\right) /\left(L^{\prime}(0) L\left(v \kappa_{d} t^{d}\right)\right) \\
J_{22}(t) & =J_{12}(t)=L^{\prime}\left(\kappa_{d} t^{d}\right) /\left(L^{\prime}(0) L\left(\kappa_{d} t^{d}\right)\right) \\
J_{. .}(t) & =J_{1 .}(t)=J_{2 .}(t)=L^{\prime}\left((1+v) \kappa_{d} t^{d}\right) /\left(L^{\prime}(0) L\left((1+v) \kappa_{d} t^{d}\right)\right) \\
I(t)= & \frac{-v}{L^{\prime}(0)(1+v)}\left[\frac{-L^{\prime}\left(v \kappa_{d} t^{d}\right)}{L\left(v \kappa_{d} t^{d}\right)}+\frac{L^{\prime}\left((1+v) \kappa_{d} t^{d}\right)}{L\left((1+v) \kappa_{d} t^{d}\right)}\right] \\
& +\frac{-1}{L^{\prime}(0)(1+v)}\left[\frac{-L^{\prime}\left(\kappa_{d} t^{d}\right)}{L\left(\kappa_{d} t^{d}\right)}+\frac{L^{\prime}\left((1+v) \kappa_{d} t^{d}\right)}{L\left((1+v) \kappa_{d} t^{d}\right)}\right]
\end{aligned}
$$

where $\kappa_{d}=m(B(0,1))$ is the volume of the unit ball in $\mathbb{R}^{d}$.

In the general case where $\left(\Lambda_{1}, \Lambda_{2}\right)$ are stationary random measures, this result holds true with $A$-weighted means replaced by expectations under the Palm distribution at 0 of $\Lambda_{2}$.

Proof. Since $X_{1}, X_{2}$ and $X$. are mixed Poisson processes with random intensity measures $\nu A m, A m$ and $(1+v) A m$ respectively, the equations for $J_{11}, J_{22}$ and $J .$. follow from theorem 6 in (Lieshout \& Baddeley, 1996) and the discussion therein. The expression for $I$ follows easily.

The reduced Palm distributions $P^{!(0,1)}, P^{!(0,2)}$ are both bivariate Cox processes with $\Lambda_{1}$ (respectively $\Lambda_{2}$ ) replaced by its $A$-weighted distribution, $\mathbb{E}_{\text {weighted }} f(A)=\mathbb{E}[A f(A)] / \mathbb{E}[A]$. The remaining identities follow.

By Lieshout \& Baddeley, 1996, th. 6, $J_{i j} \leqslant 1$ (with equality only if $A$ is constant a.s.), suggesting positive correlation between the component processes. Moreover, $J_{i j}$ is decreasing with

$$
\lim _{t \rightarrow \infty} J_{i j}(t)=\frac{\operatorname{essinf} A}{\mathbb{E} A} .
$$

By the same argument, $J_{i .}$ and $J_{\text {.. }}$ are bounded above by 1 , decreasing to essinf $A / \mathbb{E} A$. Hence, $I(t)$ converges to 0 as $t \rightarrow \infty$. Since the function

$$
s \mapsto \frac{L^{\prime}(s)}{L^{\prime}(0) L(s)}
$$

is monotonically decreasing, the terms in brackets in the expression for $I$ are both nonnegative. Thus $I$ is non-negative, confirming the positive dependence between the components. Finally, $J_{i} \leqslant J_{i i}$, confirming the positive dependence. Note that given $A$, the conditional distribution of $\left(X_{1}, X_{2}\right)$ given $X$. is that of a random labelling with

$$
\left(p_{1}, p_{2}\right)=\frac{1}{v+1}(v, 1) \text {. }
$$

(c) Board of the Foundation of the Scandinavian Journal of Statistics 1999. 
Since this distribution does not depend on $A, Y$ has the random labelling property. This is reflected in the fact that $J_{i .} \equiv J_{\text {... }}$.

\subsubsection{Balanced Cox}

An example of negative dependence is the class of balanced Cox processes (Diggle \& Milne, 1983) where

$$
\Lambda_{1}+\Lambda_{2}=v m,
$$

$m$ again denoting Lebesgue measure. Note that the superposition is always distributed as a Poisson process with intensity $\nu$.

\section{Lemma 12}

Let $\left(X_{1}, X_{2}\right)$ be a balanced Cox process on $\mathbb{R}^{d}$ with $\Lambda_{2}=$ Am, for a random variable $A$ concentrated on $(0, \nu)$ with $0<\mathbb{E} A<\nu$. Then, writing $\tilde{L}$ for the moment generating function of $v-A$,

$$
\begin{aligned}
& J_{11}(t)=\tilde{L}^{\prime}\left(\kappa_{d} t^{d}\right) /\left(\tilde{L}^{\prime}(0) \tilde{L}\left(\kappa_{d} t^{d}\right)\right) \\
& J_{22}(t)=L^{\prime}\left(\kappa_{d} t^{d}\right) /\left(L^{\prime}(0) L\left(\kappa_{d} t^{d}\right)\right) \\
& J_{12}(t)=\frac{\mathbb{E}\left[(v-A) \exp \left(-A \kappa_{d} t^{d}\right)\right]}{\mathbb{E}[v-A] \mathbb{E} \exp \left(-A \kappa_{d} t^{d}\right)} \\
& J_{21}(t)=\frac{\mathbb{E}\left[A \exp \left(-(v-A) \kappa_{d} t^{d}\right)\right]}{\mathbb{E}[A] \mathbb{E} \exp \left(-(v-A) \kappa_{d} t^{d}\right)} \\
& J_{. .}=J_{1 .}(t)=J_{2 .}(t)=1 \\
& I(t)=\frac{1}{v}\left\{\frac{-L^{\prime}\left(\kappa_{d} t^{d}\right)}{L\left(\kappa_{d} t^{d}\right)}+\frac{\tilde{L}^{\prime}\left(\kappa_{d} t^{d}\right)}{\tilde{L}\left(\kappa_{d} t^{d}\right)}\right\}
\end{aligned}
$$

as before writing $\kappa_{d}=m(B(0,1))$ for the volume of the unit ball in $\mathbb{R}^{d}$.

Proof. As the superposition is a Poisson process, $J_{\text {.. }} \equiv 1$. Again applying th. 6 of Lieshout \& Baddeley (1996), the formulae for $J_{11}$ and $J_{22}$ follow, yielding the expression for $I$.

The reduced Palm distribution $P^{!(0,2)}$ is that of a bivariate Cox process with $\Lambda_{2}$ replaced by its $A$-weighted distribution, while $P^{!(0,1)}$ is similar with $\Lambda_{1}$ governed by its $(\nu-A)$-weighted distribution. The other results follow.

\section{Using}

$$
-L^{\prime}\left(\kappa_{d} t^{d}\right) \leqslant-L^{\prime}(0) L\left(\kappa_{d} t^{d}\right)
$$

and a similar inequality with $A$ replaced by $(\nu-A)$, it is easily seen that $I \leqslant 0$, indicating negative dependence. By the discussion following Lieshout \& Baddeley (1996, th. 6), $I(t)$ decreases to (essinf $A-\operatorname{essup} A) / v$ as $t \rightarrow \infty$.

We can verify that $J_{i j} \geqslant 1$, suggesting negative correlation between the component processes. For example consider $J_{21}$. Then

$$
\begin{aligned}
& \frac{\mathbb{E}\left[A \exp \left(-(\nu-A) \kappa_{d} t^{d}\right)\right]}{\mathbb{E} A \mathbb{E} \exp \left(-(v-A) \kappa_{d} t^{d}\right)}=-\frac{\mathbb{E}\left[(\nu-A) \exp \left(-(\nu-A) \kappa_{d} t^{d}\right)\right]}{\mathbb{E} A \mathbb{E} \exp \left(-(\nu-A) \kappa_{d} t^{d}\right)}+\frac{v}{\mathbb{E} A} \\
& \geqslant-\frac{(\nu-\mathbb{E} A) \mathbb{E} \exp \left(-(v-A) \kappa_{d} t^{d}\right)}{\mathbb{E} A \mathbb{E} \exp \left(-(v-A) \kappa_{d} t^{d}\right)}+\frac{v}{\mathbb{E} A}=1 .
\end{aligned}
$$

Moreover, both $J_{12}(t)$ and $J_{21}(t)$ are monotonically increasing with limits $(v-$ 
essinf $A) /(\mathbb{E}(v-A)$ and esssup $A / \mathbb{E} A$ respectively $(t \rightarrow \infty)$. From our previous work (Lieshout \& Baddeley, 1996), $1 \equiv J_{i .} \geqslant J_{i i}$, again suggesting negative dependence between the component processes.

Turning attention to the label association, note that given $A$, the conditional distribution of $\left(X_{1}, X_{2}\right)$ given $X$. is that of a random labelling with probabilities

$$
\left(p_{1}, p_{2}\right)=\left(\frac{v-A}{v}, \frac{A}{v}\right) \text {. }
$$

Thus the allocation probabilities are random, and in general $Y$ does not satisfy the random labelling property. (This could also have been seen more directly by noting that $X$. is a Poisson process and therefore its random labelling is a bivariate Poisson process with independent components rather than the balanced Cox model we started with.) However $J_{i .}-J_{. .} \equiv 0$, which is in accordance with a random label allocation.

\subsection{Pairwise interaction Gibbs processes}

Consider a multi-type point process $Y$ with conditional intensity of the form

$$
\lambda((u, i) ; Y)=\beta_{i} \prod_{\left(x_{j}, j\right) \in Y} \gamma_{i j}\left(\left\|x_{j}-u\right\|\right)
$$

where $\beta_{1}, \ldots, \beta_{m}$ are non-negative constants and $\gamma_{i j}$ are non-negative real functions. Without loss of generality $\gamma_{i j} \equiv \gamma_{j i}$. In general, terms $\gamma_{i i}$ appear. This might be called a stationary pairwise interaction Gibbs process, cf. Ripley \& Kelly (1977). Baddeley \& Møller (1989), Ripley (1989).

Lemma 5 and equation (8) immediately yield the following.

\section{Lemma 13}

For a pairwise-interaction Gibbs process as above,

$$
\begin{aligned}
& J_{. .}(t)=\sum_{i=1}^{m} \frac{\beta_{i}}{\lambda} \mathbb{E}\left[\prod_{(x, k) \in Y} \gamma_{i k}(\|x\|) \mid X \cap \cap(0, t)=\varnothing\right] \\
& J_{i j}(t)=\frac{\beta_{i}}{\lambda_{i}} \mathbb{E}\left[\prod_{(x, k) \in Y} \gamma_{i k}(\|x\|) \mid X X_{j} \cap B(0, t)=\varnothing\right] \\
& J_{i .}(t)=\frac{\beta_{i}}{\lambda_{i}} \mathbb{E}\left[\prod_{(x, k) \in Y} \gamma_{i k}(\|x\|) \mid X, \cap B(0, t)=\varnothing\right]
\end{aligned}
$$

wherever defined. If there is finite range interaction in the sense that $\gamma_{i j}(\|x\|)=1$ for $\|x\|>r_{i j}$, the formulae above reduce to $J_{. .}(t)=\sum \beta_{i} / \lambda$ for $t \geqslant r=\max r_{i j}$ and $J_{i .}(t)$ $=\beta_{i} / \lambda_{i}$ for $t \geqslant r_{i}=\max _{j} r_{i j}$. Since in (29) the conditioning is only on no point of type $j$ in a ball around the origin, a similar reduction for $J_{i j}$ in general will not hold.

\section{Applications}

In this section we analyse two bivariate data sets and a trivariate one with a variety of correlation structures between the component processes, using empirical $I$ - and $J$-functions and Monte Carlo inference. None of the examples exhibits any obvious departures from stationarity, hence the summary statistics proposed in this paper may be used.

We consider two different null hypotheses: random labelling as described in definition 3 , and 
independence of the components $X_{i}$. To test the random labelling null hypothesis we condition on the locations and the relative frequency of the types, more specifically given a data set consisting of $n_{i}$ type $i$ events $(i=1, \ldots, m)$, the labels are permuted randomly, leaving the locations unchanged. Alternatively, we could condition on the location of the events only and sample the labels with replacement. A disadvantage of the latter is that the label probabilities are unknown-although they can be estimated by $p_{i}=n_{i} / n$ where $n_{i}$ is the number of observed $i$-events and $n$ the total number of events-and that the relative frequency of the labels is variable.

In general, non-parametric sampling from the unconditional null hypothesis of independent components is hard. For rectangular windows however, Lotwick \& Silverman (1982) proposed identifying opposite sides of the window to obtain a torus, and then translating the type 1 pattern randomly over the torus. Hence this approach is conditional on the within-component patterns rather than on the superposition locations.

Estimates of the various $F$ - and $G$-functions were computed using the Kaplan-Meier estimators of Baddeley \& Gill (1997). The corresponding $J$-functions were derived by substitution. In the case of $F$, the windows were discretized into (subsets of) rectangular pixel arrays and the distances from each pixel to the nearest data point were computed using the distance transform algorithm of Borgefors (1986). The algorithms were implemented in Splus and C. Since the $J$-function is only defined at ranges $t$ for which $F(t)<1$, and the variance of its estimator increases with increasing $t$, in the examples below we have truncated the range of values considered to those $t$ for which $F(t)<0.95$.

Within the scope of this paper we have restricted ourselves to exploratory data analysis. Of course a more formal test could be designed quite easily by considering the integrated squared difference between observed and simulated values (Besag \& Diggle, 1977). A simulation study along these lines has been performed by Thönnes and Lieshout (1998) for the univariate case, indicating that the power of tests based on $J$ is comparable to that of the more powerful of the alternatives based on $F$ or $G$. Computer simulations by Baddeley et al. (1997) suggest the surprising fact that even if more naïve and biased estimators for $F$ and $G$ are used, the tests based on $J$ retain their power.

\subsection{Beta cells in the cat retina}

Figure 1 depicts a pattern of beta-type ganglion cells in the retina of a cat recorded by Wässle et al. (1981) and kindly provided by Prof. P. J. Diggle. The window is a rectangle

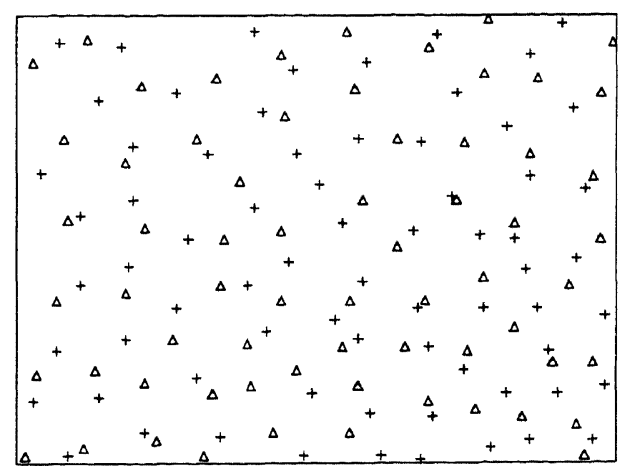

Fig. I. Sixty-five "on" $(\Delta)$ and 70 "off" $(+)$ beta cells in a cat retina. 
$(0,1) \times(0,0.7533)$ in units of approximately $1000 \mu \mathrm{m}$. Beta cells are associated with the resolution of fine detail in the cat's visual system. They can be classified anatomically as "on" $\left(X_{1}\right)$ or "off" $\left(X_{2}\right)$. Analysis of the spatial pattern provides information on the cat's visual discrimination. In particular, independence of the "on"- and "off"-components would strengthen the assumption that there are two separate channels for "brightness" and "darkness" as postulated by Hering in 1874. For details see Wässle et al. (1981). See also Diggle (1986).

Wässle et al. (1981) investigated this pattern using histograms of nearest-neighbour distances (ignoring edge effects). To test independence of the "on" and "off" patterns, a random translation of the "off"-component was superimposed upon the "on"-component, and the resulting nearest-neighbour histogram compared with the original one by a sign reversal test. They concluded that both types of beta cells form a regular lattice, which are superimposed independently.

Our analysis begins by computing estimates of the various summary statistics. The marginal nearest-neighbour distribution functions $G_{11}$ and $G_{22}$ lie below the graph of $G_{\text {.. (and similarly }}$ for $F$ ) while the cross $G_{i j}$ and $G_{i}$-functions are similar to $G_{. .}$, due to the fact that most cells

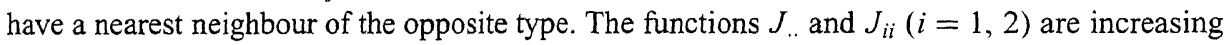
and larger than 1 , suggesting repulsion between the cells. The cross $J_{i j}$-functions are close to or above 1 , while $I$ shows slight deviations from 0 .

To investigate independence of the components we took the Lotwick-Silverman approach and repeatedly translated the first component over the torus. For each simulation, the $I$-statistic was computed. The envelopes for 99 translations and the empirical estimate of $I$ are depicted in Fig. 2. For $t$ in the range $(0,0.06)$, data curve lies between the envelopes and hence the null hypothesis is accepted. Using the statistics $J_{2}-J_{22}$ or $J_{12}$ leads to acceptance of the null hypothesis as well, see Fig. 2, as would a test based on $J_{1}-J_{11}$ or $J_{21}$. Summarizing, our results confirm conclusions of Wässle et al. (1981) thus providing evidence for the Hering postulate.

A random label allocation of types to beta cells on the other hand does not seem appropriate as a null hypothesis for testing the Hering postulate. However, for illustrative purposes we did perform the test. Since most points have a nearest neighbour of the opposite type, $J_{i}-J_{\text {.. is not }}$ a suitable test statistic, but both the cross statistics $J_{12}, J_{21}$ and $I$ lead to rejection of the random labelling hypothesis for moderate values of $t$. See Fig. 3.

A second order analysis (Hanisch \& Stoyan, 1979; Stoyan, 1984; Gavrikov \& Stoyan, 1995) by Stoyan (1995) yielded similar results. At close range, the plot of the mark correlation function $p_{12}$ (Stoyan \& Stoyan, 1994, pp. 264-265) is high compared to the plots of $p_{11}$ and $p_{22}$, before flattening down.
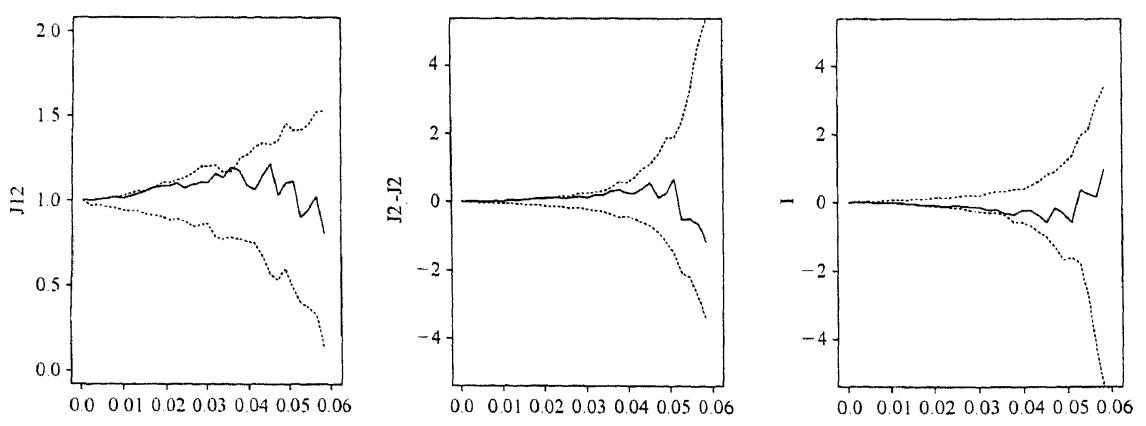

Fig. 2. Envelopes based on 99 torus translations of $X_{1}$ (dashed) and empirical $J_{12}, J_{2}-J_{22}$ and $I$ statistics for the cat retina data.

(C) Board of the Foundation of the Scandinavian Journal of Statistics 1999. 

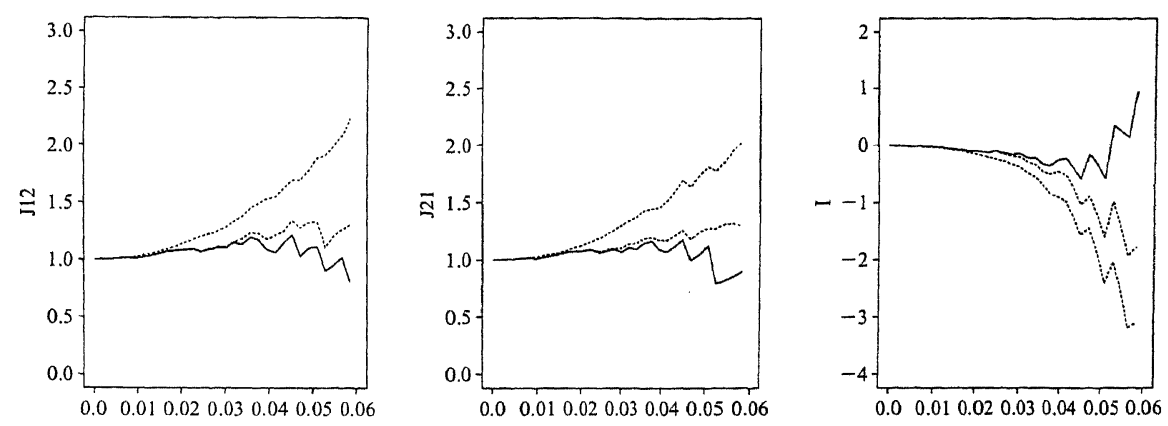

Fig. 3. Envelopes based on 99 random labellings (dashed) and empirical $J_{12}, J_{21}$ and $I$ statistics for the cat retina data.

\subsection{Myrtle trees}

Our second example is a pattern of 221 healthy and 106 diseased myrtles in a 170.5 by 213.0 metre rectangle, depicted in Fig. 4. The data set was collected by Dr H. J. Elliott and colleagues at the Forestry Commission of Tasmania (now Forestry Tasmania) and kindly supplied by Dr G. A. Kile and Prof P. J. Diggle. For further information see Packham (1994) and the references therein.

The empty spaces in three corners of the plot suggest that it would not be appropriate to treat these data as a realization of a stationary point process viewed through the rectangular frame. Instead, we have arbitrarily marked out a smaller window with a polygonal boundary as shown in Fig. 4, and computed all statistics with reference to this window.

Both components have $J_{i i}$-functions that lie below 1, suggestive of clustered behaviour. The

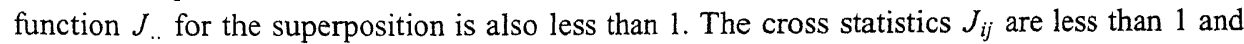
the $I$-statistic takes mostly positive values, suggesting positive dependence between the healthy and affected trees.

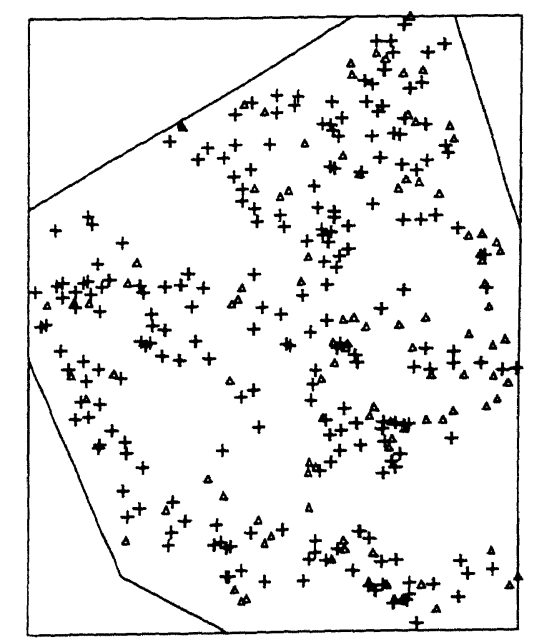

Fig. 4. Two hundred and twenty-one healthy $(+)$ and 106 diseased $(\Delta)$ myrtles, reproduced by kind permission of Dr J. Packham. 
Since the disease affects established trees, i.e. affects them after their location is fixed, it is not sensible to test for independence of the subpatterns of healthy and diseased tree locations. In any case, random toroidal shifts could not be applied since the window is not rectangular. Rather, it is appropriate to test for random labelling conditional on the tree locations.

Had we retained the original rectangular window instead of adopting the smaller polygonal boundary, the estimate of $G(t)$ for moderate values of $t$ would have remained unchanged while the estimated empty space function would have been elevated, so that the various $J$-functions would have been underestimated. However, for testing purposes this ought not to have a substantial effect, since the multiplicative bias (for fixed $t$ ) will be approximately the same. Our results support this interpretation.

The Monte Carlo envelopes over 99 simulations are given in Fig. 5. The empirical $J_{12}$, $J_{1},-J_{\text {.. }}$ and $J_{2 .}-J_{\text {.. }}$ and $I$-functions lie between the simulation envelopes for almost all values of $t$, hence for most values of $t$, the observed values of these statistics are not significant.

\subsection{Bramble canes}

Our last example concerns a trivariate pattern of bramble canes in a field 9 metres square, depicted in Fig. 6 rescaled to the unit square. The data were recorded and analysed by Hutchings (1979) and further analysed by Diggle (1981a, b, 1983) and Diggle \& Milne (1983). The canes were classified according to age as either newly emergent, one or two years old. We label these types 0,1 and 2 respectively.

All previous analyses found that the pattern of newly emergent canes exhibits clustering, which Hutchings attributes to "vigorous vegetative reproduction". Diggle (1983, sect. 7.4) also
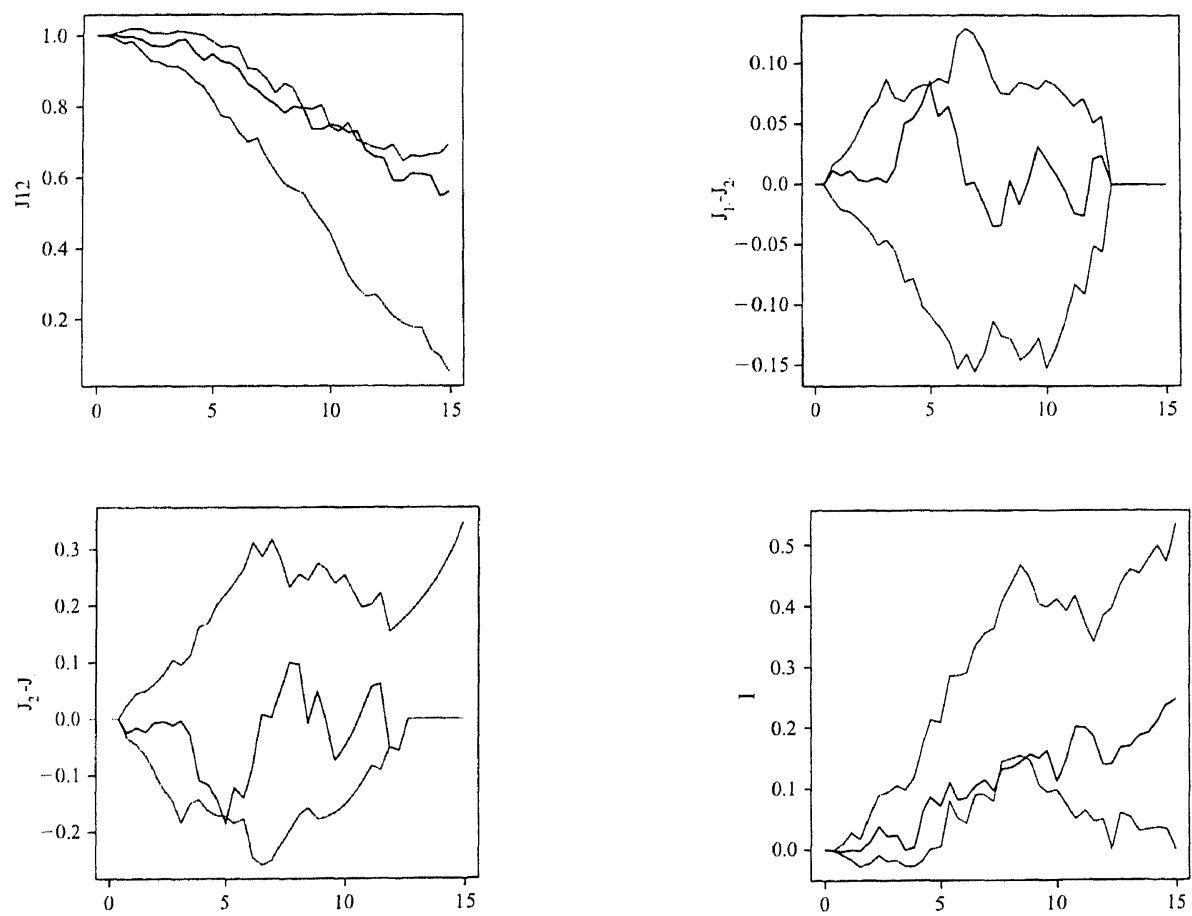

Fig. 5. Envelopes based on 99 random labellings (dashed) and empirical $J_{12}, J_{1 .}-J_{. .}, J_{2}-J_{\text {.. }}$ and $I$ statistics for the Tasmanian myrtle data. 


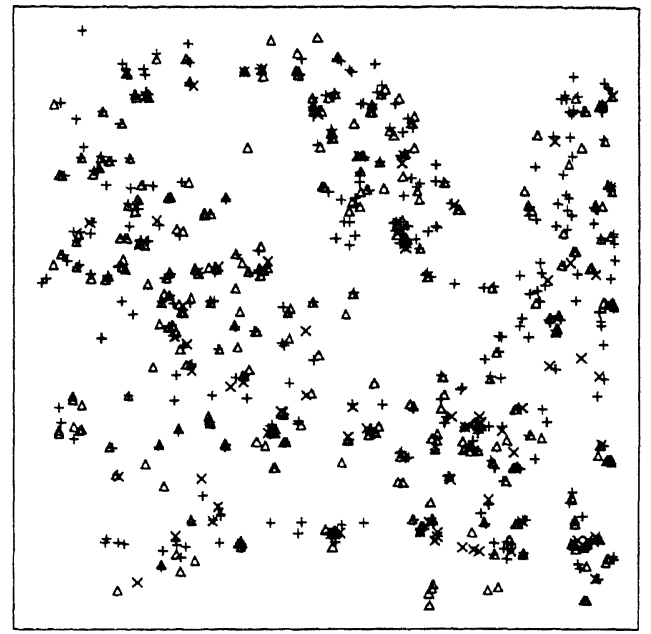

Fig. 6. Three hundred and fifty-nine newly emerging $(\Delta), 385$ one-year-old $(+)$ and 79 two-year-old $(x)$ bramble canes.

performed a bivariate analysis of the pattern of newly emerging and one-year-old canes, concluding strong positive association between them, and fitted a linked Cox process (see section 4.3.1 above). He also (Diggle, 1983, p. 117) fitted a trivariate linked Cox process to the full data set, but the fit was less satisfactory.

It should be noted that the bramble cane date is discretized; in particular there are six locations in common between $X_{0}$ and $X_{1}$, while one point contains both a newly emergent and two-year-old cane. Hence in the plots below, the estimated $I$-function for the data and the envelopes based on random labelling have an atom at 0 . When a test is based on torus translations, the common points between the components get separated almost surely, and the atom at 0 disappears.

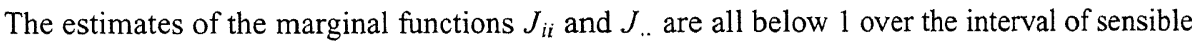
values $0 \leqslant t \leqslant 0.06$, indicating evidence for clustering. Similarly the estimated $J_{i j}$ functions are less than 1, suggesting aggregation between the points of different types. The estimates of $J_{i .}-J_{\text {.. }}$ show slight deviations from 0 ; on the other hand the estimates of $J_{i .}-J_{i i}$ are substantially below 0 throughout the interval.

Regarding the choice of test statistic, there does not seem any reason to treat the three age groups differently and we have opted for the symmetric $I$-function. Figure 7 shows the estimated $I$-function and the pointwise upper and lower envelopes from 99 simulations, obtained by applying uniformly random toroidal shifts independently to each of the three components. The I-estimate for the data lies far outside the envelopes for most of the interval, indicating emphatic evidence against the hypothesis of independence of the three types. Previous analyses have all drawn the same conclusion.

Diggle (1983, p. 114) fitted a linked Cox process to the bivariate pattern of newly emergent and one-year-old canes. The linked Cox processes envisaged (Diggle, 1983, p. 97) are more general than those treated in section 4.3.1 above, in the sense that the realized intensity functions may be non-homogeneous, but are still required to be proportional. Nevertheless, it can easily be shown that all such processes have the random labelling property (cf. Kingman, 1993). Hence it may be convenient to use a test for random labelling to assess the suitability of a linked Cox process model for these data in general, without needing to consider the specific model fitted by Diggle. 


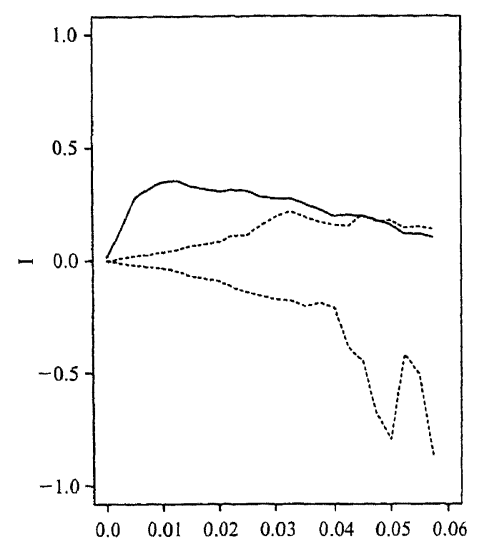

Fig. 7. Envelopes based on 99 random toroidal shifts (dashed) and empirical $I$ statistic for the bramble canes data.

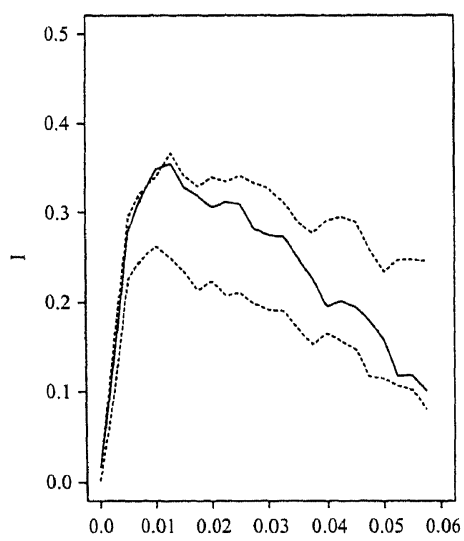

nvelopes based on 99 random labellings (dashed) and empirical $I$ statistic for the bramble canes

8 shows the estimated $I$-function and the pointwise upper and lower envelopes of the es from 99 simulations obtained by randomly permuting the age labels of the points lding the locations fixed. The estimate of $I$ now lies mostly within the simulation $\mathrm{s}$, but exceeds or lies close to the upper envelope for a considerable range of smaller $t$ suggesting that a linked Cox model does not explain the small-scale interactions torily (see Diggle, 1983, p. 117).

\section{knowledgements}

he authors are grateful to P. J. Diggle, V. Isham and G. Kile for providing data files, to J. Packham for permission to reproduce the Tasmanian myrtles data (section 5.2) and to D. Stoyan, P. J. Diggle, I. S. Molchanov, J. Møller, and A. Särkkä for valuable discussions. This research was partially carried out while the first author was at the Department of Statistics of the University of Warwick, whose support is gratefully acknowledged. Van 
Lieshout's research has also been supported by Nuffield foundation grant SCl/180/94/103 (Applications of stochastic geometry in the analysis of spatial data). Baddeley has been supported by the University of Leiden and grants from NWO and the Australian Research Council.

\section{References}

Baddeley, A. J. \& Gill, R. D. (1997). Kaplan-Meier estimators for interpoint distance distributions of spatial point processes. Ann. Statist. 25, 263-292.

Baddeley, A. J. \& Møller, J. (1989). Nearest-neighbour Markov point processes and random sets. Internat. Statist. Rev. 57, 89-121.

Baddeley, A. J., Kerscher, M., Schladitz, K. \& Scott, B. T. (1997). Estimating the J-function without edge correction. Research Report University of Western Australia, 1997/25.

Bartlett, M. S. (1975). The statistical analysis of spatial pattern. Chapman \& Hall, London.

Besag, J. \& Diggle, P. J. (1977). Simple Monte Carlo tests for spatial pattern. Appl. Statist. 26, 327-333.

Bedford, T. \& van den Berg, J. (1997). A remark on the Van Lieshout and Baddeley J-function. Adv. Appl. Probab. 29, 19-25.

Bol'shakov, I. A. (1969). Statistical problems in isolating a stream of signals from noise (in Russian). Sovyetskoye Radio, Moscow.

Borgefors, G. (1986). Distance transformations in digital images. Comput. Vision, Graphics Image Process. 34, 344-371.

Brown, T. C., Silverman, B. W. \& Milne, R. K. (1981). A class of two-type point processes. Z. Wahrsch. verw. Gebiete 58, 299-308.

Cox, D. R. \& Lewis, P. A. W. (1972). Multivariate point processes. In Proceedings of the sixth Berkeley symposium of mathematical statistics and probability, 3, 401-445. University of California Press.

Cressie, N. A. C. (1991). Statistics for spatial data. Wiley, New York.

Daley, D. J. \& D. Vere-Jones, D. (1988). An introduction to the theory of point processes. Springer-Verlag, New York.

Diggle, P. J. (1981a). Some graphical methods in the analysis of spatial point patterns. In Interpreting multivariate data, (ed. V. Barnett). Wiley, Chichester.

Diggle, P. J. (1981b). Statistical analysis of spatial point patterns. New Zealand Statist. 16, $22-41$.

Diggle, P. J. (1983). Statistical analysis of spatial point patterns. Academic Press, London.

Diggle, P. J. (1986). Displaced amacrine cells in the retina of a rabbit: analysis of a bivariate spatial point pattern. J. Neurosci. Methods 18, 115-125.

Diggle, P. J. \& Cox, T. F. (1981). On sparse sampling methods and tests of independence for multivariate spatial point processes. Bull. Internat. Statist. Inst. 49, 213-229.

Diggle, P. J. \& Milne, R. K. (1983). Bivariate Cox processes: some models for bivariate spatial point patterns. J. Roy. Statist. Soc. Ser. B 45, 11-21.

Gavrikov, V. \& Stoyan, D. (1995). The use of marked point processes in ecological and environmental forest studies. Environ. Ecol. Statist. 2, 331-344.

Griffiths, R. C. \& Milne, R. K. (1978). A class of bivariate Poisson processes. J. Multivariate Anal. 8, 380-395.

Hanisch, K.-H. \& Stoyan, D. (1979). Formulas for the second-order analysis of marked point processes. Math. Operationsforsch. Statist. Ser. Statist. 10, 555-560.

Hering, E. (1874). Zur Lehre vom Lichtsinne IV. Über die Sogenannte Intensität der Lichtempfindung und über die Empfindung des Schwarsen. Sber. Akad. Wiss. Wien, Abt. III, 69, 85-104.

Hutchings, M. J. (1979). Standing crop and pattern in pure stands of Mercurialis perennis and Rubus fruticosus in mixed deciduous woodland. Oikos 31,351-357.

Kallenberg, O. (1984). An informal guide to the theory of conditioning in point processes. Internat. Statist. Rev. 52, 151-164.

Kingman, J. F. C. (1993). Poisson processes. Oxford University Press, Oxford.

Lieshout, M. N. M. van \& Baddeley, A. J. (1996). A nonparametric measure of spatial interaction in point patterns. Statist. Neerlandica 50, 344-361.

Lotwick, H. W. \& Silverman, B. W. (1982). Methods for analysing spatial processes of several types of points. J. Roy. Statist. Soc. Ser. B 44, 406-413.

Milne, R. K. \& Westcott, M. (1972). Further results for Gauss-Poisson processes. Adv. Appl. Probab. 4, $151-176$. 
Newman, D. S. (1970). A new family of point processes characterized by their second moment properties. J. Appl. Probab. 7, 338-358.

Nguyen, X. X. \& Zessin, H. (1979). Integral and differential characterization of the Gibbs process. Math. Nachr. 88, 105-115.

Packham, J. M. (1994). Studies on myrtle wilt. PhD thesis, University of Tasmania.

Ripley, B. D. (1977). Modelling spatial patterns (with discussion). J. Roy: Statist. Soc. Ser. B 39, 172-212.

Ripley, B. D. (1989). Gibbsian interaction models. In Spatial statistics: past, present and future (ed. D. A. Griffiths). Image, New York.

Ripley, B. D. \& Kelly, F. P. (1977). Markov point processes. J. London Math. Soc. 15, 188-192.

Saxl, I. \& Rataj, J. (1996). Spherical contact and nearest-neighbour distances in Boolean cluster fields. Acta Stereol. 15, 91-96.

Stoyan, D. (1984). On correlations of marked point processes. Math. Nachr. 116, 197-207.

Stoyan, D. (1995). Personal communication.

Stoyan, D. \& Stoyan, H. (1994). Fractuls, random shapes and point fields. Wiley, New York.

Stoyan, D., Kendall, W. S. \& Mecke, J. (1995). Stochastic geometry and its applications, 2nd edn. AkademieVerlag. Berlin.

Thönnes, E. \& Lieshout, M. N. M. van (1998). A comparative study on the power of Van Lieshout and Baddeley's J-function. Research Report 334, University of Warwick.

Vincent, L. \& Jeulin, D. (1989) Minimal paths and crack propagation. Actu Stereol. 8, 487-494.

Wässle, H., Boycott, B. B. \& Illing, R.-B. (1981). Morphology and mosaic of on- and off-beta cells in the cat retina and some functional considerations. Proc. Roy: Soc. London Ser. B 212, 177-195.

Received September 1997, in final form Julv 1998

M. N. M. van Lieshout, CWI, PO Box 94079, 1090 GB Amsterdam, The Netherlands. 